\title{
Laboratory Test Results End Day
}

National Cancer Institute

\section{Source}

National Cancer Institute. Laboratory Test Results End Day. NCI Thesaurus. Code C119846.

The final day of the laboratory test. 